\title{
The Role of Inpatient Dermatology Consultations
}

\author{
Alex Sherban, BM; Matthew Keller, MD
}

\section{PRACTICE POINTS}

- Inpatient dermatologists fill knowledge gaps that often alter the diagnosis, management, and hospital course of hospitalized patients.

- Several medical specialties benefit from niche expertise of inpatient dermatologists specific to their patient population.

- Integration of inpatient dermatology consultations can prevent unnecessary hospital admissions and medication administration.
Inpatient dermatology has been shown to have profound effects on the care of hospitalized patients. However, dermatology consultations remain an underutilized resource. The purpose of this study was to demonstrate how dermatology affects the hospitalization of inpatients while highlighting the breadth of services provided. This cross-sectional retrospective study included all inpatient dermatology consultations completed at a large tertiary-care facility in an urban setting. It aimed to investigate the reasons for consultation, as well as the effects on diagnosis, management, disposition, and cutaneous condition by time of discharge. This study provides evidence supporting the integration of the dermatologist into the care of hospitalized patients by illuminating lesser-known areas of impact.

Cutis. 2021;108:193-196

$\square$ ermatology is an often-underutilized resource in the hospital setting. As the health care landscape has evolved, so has the role of the inpatient dermatologist. ${ }^{1-3}$ Structural changes in the health system and advances in therapies have shifted dermatology from an admitting service to an almost exclusively outpatient practice. Improved treatment modalities led to decreases in the number of patients requiring admission for chronic dermatoses, and outpatient clinics began offering therapies once limited to hospitals. 1,4 Inpatient dermatology consultations emerged and continue to have profound effects on hospitalized patients regardless of their reason for admission. ${ }^{1-11}$

Inpatient dermatologists supply knowledge in areas primary medical teams lack, and there is evidence that dermatology consultations improve the quality of care while decreasing cost. ${ }^{2,5-7}$ Establishing correct diagnoses, preventing exposure to unnecessary medications, and reducing hospitalization duration and readmission rates are a few ways dermatology consultations positively impact hospitalized patients. ${ }^{2,5-7,9,10}$ This study highlights the role of the dermatologist in the care of hospitalized patients at a large academic medical center in an urban setting and reveals how consultation supports the efficiency and efficacy of other services.

\section{Materials and Methods}

Study Design-This single-institution, cross-sectional retrospective study included all hospitalized patients at the Thomas Jefferson University Hospital (Philadelphia, Pennsylvania), who received an inpatient dermatology consultation completed by physicians of Jefferson Dermatology Associates between January 1, 2019, and December 31, 2019. The institutional review board at Thomas Jefferson University approved this study.

Data Collection-A list of all inpatient dermatology consultations in 2019 was provided by Jefferson Dermatology Associates. Through a retrospective chart review, data regarding the consultations were collected from the electronic medical record (Epic Systems) and recorded into the Research Electronic Data Capture system. Data on patient demographics, the primary medical team, the dermatology evaluation, and the hospital course of the patient were collected.

From the Department of Dermatology and Cutaneous Biology, Thomas Jefferson University Hospital, Sidney Kimmel Medical College, 


\section{Results}

Patient Characteristics-Dermatology received 253 inpatient consultation requests during this time period; $53 \%$ of patients were female and $47 \%$ were male, with a mean age of 55 years. Most patients were White (57\%), while $34 \%$ were Black. Five percent and $4 \%$ of patients were Asian and Hispanic or Latino, respectively (Table 1). The

\section{TABLE 1. Patient Characteristics, Reasons for Consultation, and Primary Teams Requesting Dermatology Consultations}

\begin{tabular}{|c|c|}
\hline Demographic & Patient data \\
\hline No. of patients & 253 \\
\hline \multicolumn{2}{|l|}{ Sex, n (\%) } \\
\hline Male & $118(47)$ \\
\hline Female & $135(53)$ \\
\hline Mean age, y & 55 \\
\hline \multicolumn{2}{|l|}{ Race, n (\%) } \\
\hline White & $143(57)$ \\
\hline Black & $86(34)$ \\
\hline Asian & $13(5)$ \\
\hline Hispanic or Latino & $10(4)$ \\
\hline Not reported & $1(0.4)$ \\
\hline \multicolumn{2}{|c|}{ Reason for consultation, $\mathrm{n}(\%)$} \\
\hline \multicolumn{2}{|l|}{ Assist in diagnosis } \\
\hline \multicolumn{2}{|l|}{ Assist in management } \\
\hline \multicolumn{2}{|c|}{ Full-body skin examination } \\
\hline \multicolumn{2}{|c|}{ Primary consulting team, n (\%) } \\
\hline \multicolumn{2}{|l|}{ Internal medicine } \\
\hline Emergency medicine & $36(14)$ \\
\hline Hematology/oncol & $27(11)$ \\
\hline Cardiology & $26(10)$ \\
\hline Critical care & $16(6)$ \\
\hline Neurology & $15(6)$ \\
\hline General surgery & $14(6)$ \\
\hline Gastroenterology & $7(3)$ \\
\hline Obstetrics/gynecology & $6(2)$ \\
\hline Neurosurgery & $6(2)$ \\
\hline PM\&R & $4(2)$ \\
\hline Family medicine & $2(<1)$ \\
\hline Vascular surgery & $2(<1)$ \\
\hline Cardiothoracic surgery & $2(<1)$ \\
\hline Psychiatry & $2(<1)$ \\
\hline Transplant surgery & $1(<1)$ \\
\hline Otolaryngology & $1(<1)$ \\
\hline
\end{tabular}

Abbreviation: PM\&R, physical medicine and rehabilitation. mean duration of hospitalization for all patients was 15 days, and the average number of days to discharge following the first encounter with dermatology was 10 days.

Requesting Team and Reason for Consultation-Internal medicine consulted dermatology most frequently (34\% of all consultations), followed by emergency medicine $(14 \%)$ and a variety of other services (Table 1). Most dermatology consultations were placed to assist in achieving a diagnosis of a cutaneous condition (77\%), while a minority were to assist in the management of a previously diagnosed disease (22\%). A small fraction of consultations (5\%) were to complete full-body skin examinations (FBSEs) to rule out infection or malignancy in candidates for organ transplantation, left ventricular assist devices, or certain chemotherapies. One FBSE was conducted to search for a primary tumor in a patient diagnosed with metastatic melanom

Most Common Final Diagnoses and Consultation Impact-Table 2 lists the most common final diagnosis categories, as well as the effects of the consultation on diagnosis, management, biopsies, hospitalization, and clinical improvement as documented by the primary medical provider. The most common final diagnoses were inflammatory and autoimmune (39\%), such as contact dermatitis and seborrheic dermatitis; infectious (23\%),

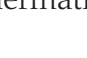

\section{TABLE 2. Most Common Final Diagnosis Categories Following Dermatology Consultation and the Effects on Care $(\mathrm{N}=253)$}

\begin{tabular}{ll}
\hline Total no. of consultations & Patient data \\
Most common diagnoses, $\mathrm{n}(\%)$ & 253 \\
\hline Inflammatory and autoimmune & $99(39)$ \\
\hline Infectious & $58(23)$ \\
\hline Drug reactions & $51(20)$ \\
\hline Other & $38(15)$ \\
\hline Vascular & $21(8)$ \\
\hline Neoplastic & $18(7)$ \\
\hline Effects of consultation, ${ }^{a} \mathrm{n}(\%)$ & $142(56)$ \\
\hline Diagnoses changed & $218(86)$ \\
\hline Management changed & $71(28)$ \\
\hline Required biopsy & $34(13)$ \\
\hline Discharged following consultation & $129(51)$ \\
\hline Improved by discharge
\end{tabular}

The effects of the consultation include all consultations regardless of reason. Single consultations may have resulted in more than 1 final diagnosis. 
such as varicella (primary or zoster) and bacterial furunculosis; drug reactions (20\%), such as morbilliform drug eruptions; vascular $(8 \%)$, such as vasculitis and calciphylaxis; neoplastic (7\%), such as keratinocyte carcinomas and leukemia cutis; and other $(15 \%)$, such as xerosis, keratosis pilaris, and miliaria rubra.

Impact on Diagnosis-Fifty-six percent of all consultations resulted in a change in diagnosis. When dermatology was consulted specifically to assist in the diagnosis of a patient (195 consultations), the working diagnosis of the primary team was changed $69 \%$ of the time. Thirty-five of these consultation requests had no preliminary diagnosis, and the primary team listed the working diagnosis as either rash or a morphologic description of the lesion(s). Sixty-three percent of suspected drug eruptions ended with a diagnosis of a form of drug eruption, while $20 \%$ of consultations for suspected cellulitis or bacterial infections were confirmed to be cellulitis or soft tissue infections.

Impact on Management-Regardless of the reason for the consultation, most consultations $(86 \%)$ resulted in a change in management. The remaining $14 \%$ consisted of FBSEs with benign findings; cases of cutaneous metastases and leukemia cutis managed by oncology; as well as select cases of purpura fulminans, postfebrile desquamation, and postinflammatory hyperpigmentation.

Changes in management included alterations in medications, requests for additional laboratory work or imaging, additional consultation requests, biopsies, of specific wound care instructions. Seventy-five percent of all consultations were given specific medication recommendations by dermatology. Most (61\%) were recommended to be given a topical steroid, antibiotic, or both. However, $45 \%$ of all consultations were recommended to initiate a systemic medication, most commonly antihistamines, antibiotics, steroids, antivirals, or immunomodulators. Dermatology recommended discontinuing specific medications in $16 \%$ of all consultations, with antibiotics being the most frequent culprit (17 antibiotics discontinued), owing to drug eruptions or misdiagnosed infections. Vancomycin, piperacillin-tazobactam, and trimethoprim-sulfamethoxazole were the most frequently discontinued antibiotics.

Dermatology was consulted for assistance in management of previously diagnosed cutaneous conditions 56 times (22\% of all consultations), often regarding complicated cases of hidradenitis suppurativa (9 cases), pyoderma gangrenosum (5 cases), bullous pemphigoid (4 cases), or erythroderma (4 cases). Most of these cases required a single dermatology encounter to provide recommendations (71\%), and $21 \%$ required 1 additional follow-up. Sixty-three percent of patients consulted for management assistance were noted to have improvement in their cutaneous condition by time of discharge, as documented by the primary provider in the medical record.

Twenty-eight percent of all consultations required at least 1 biopsy. Seventy-two percent of all biopsies were consistent with the dermatologist's working diagnosis or highest-ranked differential diagnosis, and 16\% of biopsy results were consistent with the second- or third-ranked diagnosis. The primary teams requested a biopsy 38 times to assist in diagnosis, as documented in the progress note or consultation request. Only 21 of these consultations (55\% of requests) received at least 1 biopsy, as the remaining consultations did not require a biopsy to establish a diagnosis. The most common final diagnoses of consultations receiving biopsies included drug eruptions (5), leukemia cutis (4), vasculopathies (4), vasculitis (4), and calciphylaxis (3).

Impact on Hospitalization and Efficacy-Dermatology performed 217 consultations regarding patients already admitted to the hospital, and $92 \%$ remained hospitalized either due to comorbidities or complicated cutaneous conditions following the consultation. The remaining $8 \%$ were cleared for discharge. Dermatology received 36 consultation requests from emergency medicine physicians. Fifty-three percent of these patients were admitted, while the remaining $47 \%$ were discharged from the emergency department or its observation unit following evaluation.

Fifty-one percent of all consultations were noted to have improvement in their cutaneous condition by the time of discharge, as noted in the physical examination, progress note, or discharge summary of the primary team. Thirty percent of cases remained stable, where improvement was not noted in in the medical record. Most of these cases involved keratinocyte carcinomas scheduled for outpatient excision, benign melanocytic nevi found on FBSE, and benign etiologies that led to immediate discharge following consultation. Three percent of all consultations were noted to have worsened following consultation, including cases of calciphylaxis, vasculopathies, and purpura fulminans, as well as patients who elected for palliative care and hospice. The cutaneous condition by the time of discharge could not be determined from the medical record in $16 \%$ of all consultations.

Eighty-five percent of all consultations required a single encounter with dermatology. An additional $10 \%$ required a single follow-up with dermatology, while only $5 \%$ of patients required 3 or more encounters. Notably, these cases included patients with 1 or more severe cutaneous diseases, such as Sweet syndrome, calciphylaxis, Stevens-Johnson syndrome/toxic epidermal necrolysis, and hidradenitis suppurativa.

\section{Comment}

Although dermatology often is viewed as an outpatient specialty, this study provides a glimpse into the ways inpatient dermatology consultations optimize the care of hospitalized patients. Most consultations involved assistance in diagnosing an unknown condition, but several regarded pre-existing skin disorders requiring management aid. As a variety of medical specialties requested consultations, dermatology was able to provide care to a diverse group of patients with conditions 
varying in complexity and severity. Several specialties benefited from niche dermatologic expertise: hematology and oncology frequently requested dermatology to assist in diagnosis and management of the toxic effects of chemotherapy, cutaneous metastasis, or suspected cutaneous infections in immunocompromised patients. Cardiology patients were frequently evaluated for potential malignancy or infection prior to heart transplantation and initiation of antirejection immunosuppressants. Dermatology was consulted to differentiate cutaneous manifestations of critical illness from underlying systemic disease in the intensive care unit, and patients presenting to the emergency department often were examined to determine if hospital admission was necessary, with $47 \%$ of these consultations resulting in a discharge following evaluation by a dermatologist.

Our results were consistent with prior studies ${ }^{1,5,6}$ that have reported frequent changes in final diagnosis following dermatology consultation, with $69 \%$ of working diagnoses changed in this study when consultation was requested for diagnostic assistance. When dermatology was consulted for diagnostic assistance, several of these cases lacked a preliminary differential diagnosis. Although the absence of a documented differential diagnosis may not necessarily reflect a lack of suspicion for a particular etiology, $86 \%$ of all consultations included a ranked differential or working diagnosis either in the consultation request or progress note prior to consultation. The final diagnoses of consultations without a preliminary diagnosis varied from the mild and localized to systemic and severe, further suggesting these cases reflected knowledge gaps of the primary medical team.

Integration of dermatology into the care of hospitalized patients could provide an opportunity for education of primary medical teams. With frequent consultation, primary medical teams may become more comfortable diagnosing and managing common cutaneous conditions specific to their specialty or extended hospitalizations.

Several consultations were requested to aid in management of cases of hidradenitis suppurativa, pyoderma gangrenosum, or bullous pemphigoid that either failed outpatient therapy or were complicated by superinfections. Despite the ranges in complexity, the majority of all consultations required a single encounter and led to improvement by the time of discharge, demonstrating the efficacy and efficiency of inpatient dermatologists.

Dermatology consultations often led to changes in management involving medications and additional workup. Changes in management also extended to specific wound care instructions provided by dermatology, as expected for cases of Stevens-Johnson syndrome/ toxic epidermal necrolysis, Sweet syndrome, hidradenitis suppurativa, and pyoderma gangrenosum. However, patients with the sequelae of extended hospitalizations, such as chronic wounds, pressure ulcers, and edema bullae, also benefited from this expertise.
When patients required a biopsy, the final diagnoses were consistent with the dermatologist's number one differential diagnosis or top 3 differential diagnoses $72 \%$ and $88 \%$ of the time, respectively. Only $55 \%$ of cases where the primary team requested a biopsy ultimately required a biopsy, as many involved clinical diagnoses such as urticaria. Not only was dermatology accurate in their preliminary diagnoses, but they decreased cost and morbidity by avoiding unnecessary procedures.

This study provided additional evidence to support the integration of dermatology into the hospital setting for the benefit of patients, primary medical teams, and hospital systems. Dermatology offers high-value care through the efficient diagnosis and management of hospitalized patients, which contributes to decreased cost and improved outcomes., ${ }^{2,-7,9,10}$ This study highlighted lesserknown areas of impact, such as the various specialtyspecific services dermatology provides as well as the high rates of reported improvement following consultation. Future studies should continue to explore the field's unique impact on hospitalized medicine as well as other avenues of care delivery, such as telemedicine, that may encourage dermatologists to participate in consultations and increase the volume of patients who may benefit from their care.

\section{ERENCES}

1. Madigan LM, Fox LP. Where are we now with inpatient consultative dermatology?: assessing the value and evolution of this subspecialty over the past decade. I Am Acad Dermatol. 2019;80:1804-1808. doi:10.1016/j.jaad.2019.01.031

2. Noe MH, Rosenbach M. Inpatient dermatologists-crucial for the management of skin diseases in hospitalized patients [editorial]. JAMA Dermatol. 2018;154:524-525. doi:10.1001/jamadermatol.2017.6195

3. Strowd LC. Inpatient dermatology: a paradigm shift in the management of skin disease in the hospital. Br J Dermatol. 2019;180:966-967. doi:10.1111/bjd.17778

4. Kirsner RS, Yang DG, Kerdel FA. The changing status of inpatient dermatology at American academic dermatology programs. J Am Acad Dermatol. 1999;40:755-757. doi:10.1016/s0190-9622(99)70158-1

5. Kroshinsky D, Cotliar J, Hughey LC, et al. Association of dermatology consultation with accuracy of cutaneous disorder diagnoses in hospitalized patients: a multicenter analysis. JAMA Dermatol. 2016;152:477-480. doi:10.1001/jamadermatol.2015.5098

6. Ko LN, Garza-Mayers AC, St John J, et al. Effect of dermatology consultation on outcomes for patients with presumed cellulitis. JAMA Dermatol. 2018;154:529-533. doi:10.1001/jamadermatol.2017.6196

7. Li DG, Xia FD, Khosravi $\mathrm{H}$, et al. Outcomes of early dermatology consultation for inpatients diagnosed with cellulitis. JAMA Dermatol. 2018;154:537-543. doi:10.1001/jamadermatol.2017.6197

8. Milani-Nejad N, Zhang M, Kaffenberger BH. Association of dermatology consultations with patient care outcomes in hospitalized patients with inflammatory skin diseases. JAMA Dermatol. 2017;153:523-528. doi:10.1001/jamadermatol.2016.6130

9. Imadojemu S, Rosenbach M. Dermatologists must take an active role in the diagnosis of cellulitis. JAMA Dermatol. 2017;153:134-135. doi:10.1001/jamadermatol.2016.4230

10. Hughey LC. The impact dermatologists can have on misdiagnosis of cellulitis and overuse of antibiotics: closing the gap. JAMA Dermatol. 2014;150:1061-1062. doi:10.1001/jamadermatol.2014.1164

11. Ko LN, Kroshinsky D. Dermatology hospitalists: a multicenter survey study characterizing the infrastructure of consultative dermatology in select American hospitals. Int J Dermatol. 2018;57:553-558. doi:10.1111/ijd.13939 